\title{
Cardiac myoglobin participates in the metabolic pathway of selenium in rat
}

\author{
Eriko Hori, ${ }^{a}$ Sakura Yoshida, ${ }^{a}$ Takeshi Fuchigami, ${ }^{a}$ Mamoru Haratake ${ }^{* b}$ and Morio \\ Nakayama*a
}

${ }^{a}$ Graduate School of Biomedical Sciences, Nagasaki University, 1-14 Bunkyo-machi, Nagasaki852-8521, Japan._E-mail:morio@nagasaki-u.ac.jp

${ }^{\mathrm{b}}$ Faculty of Pharmaceutical Sciences, Sojo University, 4-22-1 Ikeda, Kumamoto 860-0082, Japan. E-mail: haratake@ph.sojo-u.ac.jp

An essential micronutrient selenium deficiency is a leading-cause of cardiovascular diseases. The heart is continuously beating for blood delivery to the entire body, which requires a high-energy demand. The adult heart normally obtains $50-70 \%$ of its adenosine 5'-triphosphate from fatty acid $\beta$-oxidation. An increase in the fatty acid oxidation activity induces the generation of larger amounts of by-products (reactive oxygen species, ROS) from mitochondrial oxidative phosphorylation. Selenium-dependent glutathione peroxidases play the critical role for removal of the ROS, especially organic hydroperoxides, from the heart. The definitive transport and/or detailed metabolic pathways from the selenium-source compounds to the selenoproteins in the heart still remain unclear. We explored the selenium-binding proteins in the rat cardiac cell lysate using its reactive metabolic intermediate selenotrisulfide (STS) and MALDI TOF-mass spectrometry. Several proteins with the free cysteine (Cys) thiol were found to be reactive with STS through the 
thiol-exchange reaction. The most distinctive Cys-containing protein in the cardiac cell lysate was identified as myoglobin $(\mathrm{Mb})$ from a rat protein database search and tryptic fragmentation experiments. When separately examined in selenium-adequate rats, selenium-binding to the cardiac $\mathrm{Mb}$ was verified by the selenium-specific fluorometry. Cardiac Mb was thought to participate in the selenium metabolic pathway in the heart.

\section{Significance to metallomics}

Selenium is a critical element for the antioxidant defense against the oxidative damage caused by respiration. A better understanding of the systemic delivery mechanisms and cellular metabolic pathway of selenium from selenium-source compounds is of significance from the viewpoints of human medical treatments and toxicology. We demonstrated that cardiac myoglobin participates in the selenium metabolism in the rat heart.

\section{Introduction}

Dietary selenium is an essential micronutrient for humans and other mammals. ${ }^{1}$ Unlike other essential micronutrients, such as zinc and iron, selenium is directly incorporated into polypeptide chains as selenol-L-cysteine (SeCys), the 21st proteinogenic amino acid encoded by the SeCys insertion uracil-guanine-adenine codon. Twenty-five SeCys-containing proteins (selenoproteins) have been identified in humans and their physiological functions are being investigated. ${ }^{2} \quad$ Glutathione peroxidases (GPxs) are the best characterized selenoproteins, which catalyze the reduction of organic and inorganic peroxide species $(\mathrm{R}-\mathrm{OOH})$ to alcohols $(\mathrm{R}-\mathrm{OH})$ at their active center SeCys residue. ${ }^{3}$ Selenium is the critical 
element for the antioxidant defense against the oxidative damage inevitably caused by respiration.

This micronutrient is acquired through the consumption of various foods including grains, fish and meat. ${ }^{4}$ The selenium content in terrestrial foods is solely dependent on the selenium content of the soil where plants and/or animal feeds are grown. Human selenium deficiency occurs, but is rare, in several areas of China and Russia and, to a lesser extent in some parts of Europe, where the selenium content of the soil is very low and foods grown locally are the predominant sources of nutrients. Keshan disease (KD) is a cardiomyopathy endemic in China and the KD incidence is positively associated with the nutritional status of selenium. ${ }^{5}$ As a consequence, the expression and activity of GPxs in the KD patients' heart is known to be lower than that in healthy subjects. ${ }^{6}$ Such a selenium deficiency brings about myocardial disease, muscle weakness, nail deformation, etc. A meta-analysis of several observational studies has suggested a significant inverse association between the risk of coronary heart disease and selenium status, although intervention studies with selenium supplementation have not shown a protective effect against cardiovascular disease. ${ }^{7-9}$ Sodium selenite and selenomethionine supplements are commonly used to boost the selenium concentrations. ${ }^{10,11}$ Therefore, a better understanding of the systemic delivery mechanisms of selenium from selenium-source compounds is of significance from the viewpoints of human medical treatments and toxicology.

Selenotrisulfide (R-S-Se-S-R, STS) is one of the key metabolic intermediates of selenium. $^{12}$ The presence of STS species with low molecular-mass thiols (glutathione) has been documented in biological systems by a modern mass spectrometric technique. ${ }^{13}$ Under physiological conditions, a nucleophilic thiolate $\left(\mathrm{R}-\mathrm{S}^{-}\right)$of the L-cysteine residue (Cys) 
preferably attacks the more electrophilic selenium atom of STS than its sulfur atoms. This reaction is a bimolecular nucleophilic substitution reaction $\left(\mathrm{SN}_{2}\right)$. STS allows reactions with biogenic proteins with the free Cys thiol(s), e.g., hemoglobin and albumin, through the thiol-exchange reaction. ${ }^{14-16}$ When a thiolate attacks an STS-containing species, the leaving group would be the thiol with a higher pKa value. The resulting selenium-bound protein species can often participate in the subsequent transport and/or metabolic pathways of the selenium. $^{17,18}$ In biological processes, the thiol-exchange reaction is the basis for the mechanism of action of many enzymes. ${ }^{19}$

Based on a comprehensive analysis using an STS species, selenium from selenious acid was found to bind to the liver fatty acid-binding protein (LFABP) through its only thiol in the mouse and rat (Fig. 1). ${ }^{20}$ This finding was consistent with the previous results from an independent profiling experiment using a combination of the radioactive tracer $\left(\mathrm{H}^{75} \mathrm{SeO}_{3}\right)$ and Western blotting techniques. ${ }^{21}$ In the present study, our analytical methodology was extended for profiling the selenium-binding proteins in the heart. Selenium deficiency is the leading-cause of cardiovascular diseases, as previously mentioned. The heart is continuously beating for blood delivery to the entire body, which requires a high-energy demand, as does the brain. The adult heart normally obtains $50-70 \%$ of its adenosine 5'-triphosphate from fatty acid $\beta$-oxidation. ${ }^{22}$ An increase in the fatty acid oxidation activity induces the generation of larger amounts of by-products (reactive oxygen species, ROS) from the mitochondrial oxidative phosphorylation. Consequently, selenium-dependent GPxs play a critical role in the removal of the ROS, especially organic hydroperoxides, from the heart. ${ }^{23}$ However, definitive transport and/or detailed metabolic pathways from the selenium-source compounds to the selenoproteins in the heart still remain unclear. 


\section{Experimental}

\section{Materials}

Selenious acid (SA), trifluoroacetic acid, $N$-ethylmaleimide (NEM), glutathione in the reduced form (GSH) and trypsin (from porcine pancreas, 1000-2000 units $\mathrm{mg}^{-1}$, salt free) were purchased from Nacalai Tesque, Inc. (Kyoto, Japan). L-Penicillamine (Pen) was purchased from Tokyo Chemical Industry Co., Ltd. (Tokyo, Japan) and 2,3-diaminonaphthalene (DAN) was from Dojindo Molecular Technologies, Inc. (Kumamoto, Japan). Bovine serum albumin (BSA) was from Wako Pure Chemical Industries, Ltd. (Osaka, Japan). Matrix-assisted laser desorption ionization time of flight-mass spectrometry (MALDI TOF-MS)-grade sinapic acid and 5,5'-dithiobis(2-nitrobenzoic acid) (DTNB) were from the Sigma Co. (St. Louis, MO, U.S.A.). The dialysis tube Spectra/Por Membrane (regenerated cellulose, molecular mass cutoff; 6-8 kDa) was from Spectrum Laboratories, Inc. The atomic absorption spectrometry-grade selenium standard solution [1000 ppm as selenium (IV) dioxide in $0.5 \mathrm{M}$ nitric acid] was from Kanto Chemical Co., Inc. (Tokyo, Japan). The molecular mass calibration was carried out using the \#206355 Protein Calibration Standard that is composed of insulin, ubiquitin I, cytochrome $c$ and myoglobin from Bruker Daltonics, Inc. (Bremen, Germany). The water used throughout this study $(>18 \mathrm{M} \Omega \mathrm{cm})$ was generated by a Milli-Q Biocel system (Millipore Corp., Billerica, MA, USA). All other chemicals were of commercial reagent or special grade and used as received.

\section{Animal}

Three-week old male Wistar rats were purchased from CLEA Japan, Inc. (Tokyo, Japan). 
All experiments with live animals were performed in compliance with the guidelines of the Nagasaki University on Animal Care and Use, and the institutional committee approved the experimental protocols. Rats were housed on a $12 \mathrm{~h}$ light/ $12 \mathrm{~h}$ dark schedule at $25 \pm 2{ }^{\circ} \mathrm{C}$ and $60 \%$ relative humidity, and were freely given deionized water.

\section{Synthesis of PenSSeSPen}

L-Penicillamine selenotrisulfide (PenSSeSPen) was synthesized according to a previously described procedure. ${ }^{14,24}$ Briefly, a $4 \mathrm{mM}$ Pen solution was poured into a $1 \mathrm{mM}$ SA solution and the mixture was allowed to react with stirring for $3 \mathrm{~h}$ at room temperature and left for another $20 \mathrm{~h}$ at $4{ }^{\circ} \mathrm{C}$. The resulting snow-white precipitate was isolated and carefully washed twice with cold water, then washed twice with cold methanol. The obtained material was dried in vacuo at room temperature for $24 \mathrm{~h}$ or longer, then stored in a desiccator until used. PenSSeSPen was dissolved in Milli-Q water just before each experiment and chromatographically checked. Elemental analysis (\%), calcd for $\mathrm{C}_{10} \mathrm{H}_{20} \mathrm{~N}_{2} \mathrm{O}_{4} \mathrm{~S}_{2} \mathrm{Se}$ : C 31.97, H 5.33, N 7.46, Se 21.04, found: C 31.13, H 5.86, N 7.24, Se 21.89. $[\alpha]_{\mathrm{D}},+7.07 . \quad \lambda_{\max }$ in deionized water $266 \mathrm{~nm}\left(\varepsilon_{\mathrm{mM}}: 1.47\right)$. MALDI TOF-MS (positive ion mode), calcd for $\mathrm{C}_{10} \mathrm{H}_{20} \mathrm{~N}_{2} \mathrm{O}_{4} \mathrm{~S}_{2}{ }^{80} \mathrm{Se} 376.2$, found $m / z$ 376.3.

\section{Preparation of rat heart cell lysate and reaction with PenSSeSPen}

A male Wistar rat (3-4 weeks old, body weight $\approx 120$ g) was sacrificed under ether anesthesia and its heart removed. The isolated heart was thoroughly rinsed with $1 \mathrm{mM}$ Tris-HCl buffer (pH 7.4). The heart tissue was homogenized by a Polytron PT1200E (Kinematica AG, Luzern, Switzerland), then sonicated at the acoustic power level of $20 \mathrm{~W}$ by a probe-type 
sonicator 250D (Branson Ultrasonic Corp., Danbury, CT, USA). The heart tissue suspension was transferred to a polycarbonate centrifuge tube $(38 \times 102 \mathrm{~mm})$. Subsequently, the heart cell lysate was obtained by centrifugation in a rotor TYPE45Ti using an Optima L-80 Ultracentrifuge (Beckman Coulter Inc., Indianapolis, IN, USA) at $20000 \mathrm{rpm}$ for $60 \mathrm{~min}$ at 4 ${ }^{\circ} \mathrm{C}$. The obtained supernatant was used in the experiments. For further purification, the diluted lysate was passed through a regenerated Amicon cellulose membrane (molecular mass cutoff: $30 \mathrm{kDa}$, Millipore Corp., Billerica, MA, USA) after dialysis. PenSSeSPen solutions of the desired concentrations were dissolved in Milli-Q water just before mixing with the heart cell lysate. The PenSSeSPen solutions were combined with the rat heart cell lysate, then the mixture was allowed to react for 10 min at $37^{\circ} \mathrm{C}$.

\section{Determination of selenium and protein thiol concentrations}

The cell lysate specimens were dialyzed six times against $1 \mathrm{mM}$ Tris- $\mathrm{HCl}$ buffer $(\mathrm{pH} 7.4)$ using a Spectra/Por Membrane at $4{ }^{\circ} \mathrm{C}$ to remove the low-mass thiols and the remaining PenSSeSPen. The protein concentrations in the specimens after dialysis were measured by the Lowry method using bovine serum albumin as the standard. ${ }^{25}$ The selenium concentrations were fluorometrically determined using DAN after acid digestion with a 1:4 mixture by volume of perchloric acid and nitric acid. ${ }^{26}$ The selenium standard solution was employed as the standard material for preparation of the calibration curve. The fluorescence intensity was recorded by a FP-6600 fluorometer (Jasco Corporation, Tokyo, Japan) (excitation wavelength: $375 \mathrm{~nm}$, emission wavelength: $520 \mathrm{~nm}$ ). The protein thiol concentrations were colorimetrically determined using DTNB. ${ }^{27}$ An equal volume of a 1 $\mathrm{mM}$ DTNB solution in a $1 \mathrm{mM}$ Tris- $\mathrm{HCl}$ buffer ( $\mathrm{pH}$ 7.4) was added to the cell lysate samples. 
After incubation for $30 \mathrm{~min}$, the absorbance at $450 \mathrm{~nm}$ was monitored by a V-660 spectrometer (JASCO Corp.). GSH was used as the standard compound for preparation of the calibration curve.

\section{Determination of cellular glutathione peroxidase activity}

The cell lysate was combined with sodium azide $(0.63 \mathrm{mM})$, a glutathione reductase solution (0.63 unit), a reduced glutathione solution $(0.63 \mathrm{mM})$ and a NADPH solution $(2.34 \mathrm{mM})$ in $0.05 \mathrm{M}$ phosphate buffer $(\mathrm{pH}$ 7.4). The reaction was initiated by the addition of a hydrogen peroxide solution $(3.14 \mathrm{mM})$. Absorbance at $340 \mathrm{~nm}$ due to the NADPH was recorded every $10 \mathrm{~s}$ just after mixing by inversion. The GPx activity was calculated using the following equation (1) as $\mu$ moles of NADPH oxidized per minute, where $\triangle A$ SAM is the decrease in the absorbance at $340 \mathrm{~nm}$ of the sample solutions between 10 and $70 \mathrm{~s}$ after the addition of the substrates, $\triangle A$ BLK is the decrease in absorbance at $340 \mathrm{~nm}$ per minute of the solutions using saline instead of the sample solutions, 20.6 is the dilution factor, $\varepsilon_{\mathrm{mM}}$ is the extinction coefficient for the $1 \mathrm{mM}$ NADPH solution $\left(6.22 \mathrm{mM}^{-1} \mathrm{~cm}^{-1}\right)$, and $c$ is the protein content $(\mu \mathrm{g})^{28}$

$$
\text { cGPx activity }=\left(\Delta A_{\mathrm{BLK}}-\Delta A_{\mathrm{BLK}}\right) \times 20.6 / \varepsilon_{\mathrm{mM}} / \mathrm{c}
$$

\section{Sodium dodecyl sulfate-polyacrylamide gel electrophoresis}

After measurement of the protein concentration by the Lowry method, the samples were dissolved in a mixture of $1 \%$ SDS, $0.01 \mathrm{M}$ Tris- $\mathrm{HCl}(\mathrm{pH} 7.4)$ and $20 \%$ glycerin. The samples containing $5 \mu \mathrm{g}$ of protein were electrophoresed on a $15 \%$ polyacrylamide gel e-PAGEL (ATTO Corp., Tokyo, Japan) with an electrophoresis buffer (0.1\% SDS, 0.1 M Tris, 
$0.05 \mathrm{M}$ tricine) at $20 \mathrm{~mA}$ plate ${ }^{-1}$ for $1.5 \mathrm{~h}$ using a pageRun AE-6531M (ATTO Corp.). The molecular mass calibration was carried out using Precision Plus Protein Standards that contain ten bands of 10-200 kDa (Bio-Rad Lab., CA, USA). The locations of the proteins in the gels were determined by silver staining (EzStain Silver, ATTO Corp.).

\section{MALDI TOF-MS (Matrix-assisted laser desorption ionization time of flight-mass spectrometry)}

The sample solutions were mixed with an equal volume of matrix solution [saturated sinapic acid or $\alpha$-cyano-4-hydroxy cynnamic acid (CHCA) in $0.1 \%$ trifluoroacetic acid and $34 \%$ acetonitrile for the specimens], and an aliquot was applied on an AnchorChip target matrix thin layer. Sinapic acid and CHCA were used for the mass spectrometric analysis of the protein species in the cell lysate and peptide fragments after trypsin-digestion, respectively. The mass spectra were acquired in the linear positive ion mode using an Ultraflex MALDI TOF/TOF-MS (Bruker Daltonics, Inc.). Each spectrum was produced by accumulating data from 5000 consecutive laser shots. The molecular mass calibration was carried out using the \#206355 Protein Calibration Standard. Both the PenSSeSPen- and NEM-reactive protein species were subjected to a database search using the Protein Information Resource (http://www-nnbrf.geogetown.edu/pirwww/). The candidate proteins were identified by the tryptic fragment mass data from the MS-Digest program of Protein Prospector (http://prospector.ucsf.edu/prospector/mshome) (Fig. S1). The heart cell lysate samples in the molecular mass range of interest were separated by dialysis using a regenerated cellulose membrane (molecular mass cutoff $6-8 \mathrm{kDa}$ ), then ultrafiltration through a regenerated cellulose membrane (molecular mass cutoff; $30 \mathrm{kDa}$ ) before the tryptic digestion. 


\section{Statistical analysis}

All data are presented as the mean and standard deviation (SD). Statistical analyses were performed using PRISM 4 (GraphPad Software, Inc., La Jolla, CA, USA). Statistically significant differences between the adequate and selenite-fed group were determined by the two-tailed unpaired $t$-test. Comparisons were considered to be statistically significant at $P<$ 0.05 .

\section{Results and discussion}

Most of the selenotrisulfide (R-S-Se-S-R, STS) species formed with low molecular-mass thiols, such as glutathione (GSH) and coenzyme A, are highly labile at the physiological $\mathrm{pH}$ in vitro. Thus, it is hardly possible to chemically isolate the pure STS species. However, penicillamine STS (PenSSeSPen) (Fig. 1 top) with L-penicillamine (L- $\beta, \beta$-dimethylcysteine, Pen) is quite stable without any chemical degradation for $24 \mathrm{~h}$ or longer under the physiological conditions. ${ }^{24}$ The elemental analysis results of PenSSeSPen used in this study were in good agreement with the calculated value. When the PenSSeSPen sample was subjected to a MALDI TOF-mass analysis, its mass spectrum with the characteristic selenium isotope pattern was consistent with the calculated one.

To determine the reactivity of STS to the cytosolic protein species, the rat cardiac cell lysate was incubated with PenSSeSPen for $10 \mathrm{~min}$ at $37^{\circ} \mathrm{C}$, then the protein-bound selenium amount in the resulting cell lysate was determined by the 2,3-diaminonaphthalene (DAN) method subsequent to acid digestion after removing the low molecular-mass compounds such 
as GSH and the unreacted PenSSeSPen. The amount of selenium bound to the proteins increased with an increase in the PenSSeSPen concentration and reached a plateau $(\approx 20 \mu \mathrm{mol}$ $\mathrm{g}^{-1}$-protein) (Table 1), which was almost equal to that of the protein thiol amount in the cardiac cell lysate $\left(20.98 \mu \mathrm{mol} \mathrm{g}^{-1}\right.$-protein). Thus, with its increasing concentration, PenSSeSPen was basically capable of reacting with almost all the protein thiols that were detected by the DTNB method.

To clarify the protein species that were preferably reacted with PenSSeSPen, the cardiac cell lysates before and after the reaction with PenSSeSPen were subsequently subjected to a MALDI TOF-mass spectrometric analysis (Table 2). The mass spectral peaks of the thiol-containing proteins in the cell lysate were specified by the molecular mass gain in $\mathrm{m} / \mathrm{z}$ by 125 after chemical derivatization (alkylation) with $N$-ethylmaleimide (NEM) in comparison to that in the mass spectrum before the reaction. More than ten kinds of peaks were detected as thiol-containing proteins, and three of them gave multiple NEM-adducts. After the reaction with PenSSeSPen, the molecular-mass of seven thiol-containing proteins that were reacted with NEM resulted in an $\mathrm{m} / \mathrm{z}$ gain of 226. Such an increase in the molecular mass due to the reaction with PenSSeSPen corresponds to that of the selenenyl-Pen (-SeSPen) moiety. All the protein peaks reactive with PenSSeSPen contain the free thiol, indicating that PenSSeSPen could possibly react with the seven protein species through a thiol-exchange reaction to form the asymmetrical STS $(\mathrm{R}-\mathrm{Cys}-\mathrm{SH}+$ PenSSeSPen $\rightarrow \mathrm{R}-$ Cys-SSeSPen + Pen-SH). The intensity of the $m / z 17026$ species was the highest among the seven PenSSeSPen-reactive proteins (Fig. 2). Moreover, to verify the reactivity of PenSSeSPen with the $\mathrm{m} / \mathrm{z} 17026$ species, the cardiac cell lysates treated with increasing PenSSeSPen concentrations were analyzed by MALDI TOF-mass spectrometry. The $m / z$ 
17026 species was reacted with PenSSeSPen in a concentration-dependent manner (Fig. 3).

The intensity of the peak at $\mathrm{m} / \mathrm{z} 17026$ gradually decreased with the increasing PenSSeSPen concentration, while the intensity of the SeSPen-adduct peak at $m / z 17252$ increased in an inverse fashion.

A database search using the Protein Information Research suggested rat myoglobin $(\mathrm{Mb})$ as the protein species with $\mathrm{m} / \mathrm{z}$ 17026. The Mb molecule consists of 153 amino acid residues and has the only free thiol at Cys66 (Fig. 4). The m/z 17026 species was separated from the cardiac cell lysate by ultrafiltration and dialysis techniques. The purified cell lysate sample was subjected to an SDS-PAGE analysis followed by silver staining, together with that before purification (Fig. 5B). A silver-stained single band was only detected slightly above the 15 $\mathrm{kDa}$ molecular-mass marker band for the purified cell lysate sample (Fig. 5B, lane b). Purification of $\mathrm{Mb}$ from rat heart cell lysate was also confirmed by gel permeation chromatography (Fig. S1). The purified cell lysate sample was further digested with trypsin and the resulting material was subjected to a MALDI TOF-mass spectrometric analysis. The characteristic tryptic fragments from the rat Mb [Val17-Lys34 (molecular mass calcd 1913, found 1912.6), Asn48-Lys56 (calcd 1124, found 1127.6), Gly80-Lys96 (calcd 1788, found 1786.1), Tyr103-Lys116 (calcd 1695, found 1695.6), Tyr119-Lys133 (calcd 1505, found 1505.2), Ala146-Gly153 (calcd 942, found 942.4)] were clearly detected from the cell lysate sample after the enzyme digestion (Fig. S2). The molecular ion peak at $\mathrm{m} / \mathrm{z} 17026$ completely disappeared from the mass spectrum of the trypsin-digested cell lysate. Based on all these results together, the $\mathrm{m} / \mathrm{z} 17026$ protein species that reacted with PenSSeSPen was identified as the rat $\mathrm{Mb}$.

To verify the results from our model experiments using a non-biogenic STS, the reaction 
of glutathione STS (GSSeSG) with Mb in the cardiac cell lysate was evaluated by MALDI TOF-mass spectrometry. GSSeSG is thought to be one of the most typical STS species to be generated in vivo. GSH and SA were added to the cardiac cell lysate, then the mixture was allowed to react at $37^{\circ} \mathrm{C}$. When only SA was added to the cell lysate, the appearance of a peak corresponding to the SeSG-adduct of $\mathrm{Mb}$ at $\mathrm{m} / \mathrm{z} 17411$ (gain in $\mathrm{m} / \mathrm{z}$ by 385) was not remarkable, probably due to low GSH concentration, and not enough to generate GSSeSG (Fig. 6B). The addition of SA together with GSH at the appropriate concentration resulted in the generation of the distinctive peak of the SeSG-adduct of Mb (Fig. 6C). Mb was thought to be capable of forming the STS species through the thiol-exchange reaction with GSSeSG. We also tried to detect Mb-SeSG species from in vivo rat heart cell lysate samples by MALDI TOF mass spectrometry, but were not successful due to its low concentration.

The cardiac $\mathrm{Mb}$ in conventional 6-week old male selenium-adequate rats was isolated, then subjected to the determination of selenium by the DAN method subsequent to acid digestion. Selenium was practically detected from the isolated cardiac Mb in rats; its content was $6.93 \pm 2.74 \mu \mathrm{gSe} \mathrm{g}^{-1}$-protein (mean $\pm \mathrm{SD}, n=4$ ). Because of its very small fraction, selenium-bound $\mathrm{Mb}$ could not be directly detected by MALDI TOF-mass spectrometry. To obtain basic information about the selenium binding to the cardiac $\mathrm{Mb}$, additional SA plus a regular diet containing a nutrition level of selenium was orally administrated to the rats twice-a-day for 7 days at $1.5 \mu \mathrm{gSe} \mathrm{kg}^{-1}$-body weight, and the amount of selenium bound to the cardiac $\mathrm{Mb}$ was determined. The amount of selenium bound to the cardiac $\mathrm{Mb}$ after the $\mathrm{SA}$ administration was $6.10 \pm 0.28 \mu \mathrm{gSe} \mathrm{g}^{-1}$-protein (mean $\pm \mathrm{SD}, n=3$ ), which was almost comparable to the value for the $\mathrm{Mb}$ of the non-administered (regular diet only) rat. The additional SA-administration resulted in an increase in total selenium content in the heart 
$\left(0.26 \pm 0.056\right.$ to $0.51 \pm 0.16 \mu g \mathrm{ge} \mathrm{g}^{-1}$-heart) (Table S1). The cellular GPx activity in the heart also significantly increased in comparison to the non-administered rats (Fig. 7B). Therefore, $\mathrm{Mb}$ was thought to act as a selenium relay point for the synthesis of the selenoproteins in the heart. In the dietary selenium-depleted state, the selenium concentration in the heart decreases less in comparison to those in the blood and liver. ${ }^{24,29}$ Taking this fact into consideration, the selenium-binding to Mb implies a store of selenium in the heart, as it acts on molecular oxygen. In the same rat experiments, the selenium concentration in the liver also increased by the additional SA administration of $(0.60 \pm 0.14$ to $2.95 \pm 0.47 \mu \mathrm{gSe}$ $\mathrm{g}^{-1}$-liver) (Table S1), whereas the cellular GPx activity in the hepatic cell lysate did not markedly change (Fig. S3). Selenium-dependent GPxs have five isoforms that are different in their distribution pattern and affinity to peroxide substrates. About half of the selenium in the liver belongs to the cellular GPx (GPx1). The dietary selenium-depletion induces a higher degree of decrease in the selenium concentration in the liver compared to that in the heart. $^{24}$ The expression level of GPx3 and GPx4 in the heart is higher than those in the other organs and tissues. ${ }^{30}$ The expression level of the GPx isoforms could possibly be responsible for the changes in the cellular GPx activity in the cardiac and hepatic cell lysates after the SA administration.

$\mathrm{Mb}$ is similar to hemoglobin $(\mathrm{Hb})$ in its structure and function; they both bind with molecular oxygen through the heme. Whereas $\mathrm{Hb}$ transports oxygen from the lungs to the other peripheral tissues, the $\mathrm{Mb}$ function is to store oxygen and release it under its depletion in muscle tissues. In aquatic mammals, the $\mathrm{Mb}$ concentrations are $\approx 10$-fold higher than those of terrestrial mammals. ${ }^{31}$ Human $\mathrm{Mb}$ also has a free thiol at Cys110 (Fig. S4). ${ }^{32}$ It was only reported that selenocyanate could be a probe of the local electrostatic environment 
in the heme pocket of horse $\mathrm{Mb}^{33}$ This is the first report that the free thiol of Mb can react with selenium compounds. Because increased fatty acid oxidation causes the increased generation of the ROS, GPxs play important roles as antioxidative defense systems. Especially, GPx4 is the only antioxidative enzyme that can directly reduce phospholipid hydroperoxides generated in biological membranes, hence, this enzyme also plays a critical role in the developmental process and biological activity. ${ }^{34-36}$ Both selenium and $\mathrm{Mb}$ are physiologically important factors for maintaining the heart-beat system. The biological significance of selenium-binding to Mb should be further explored.

In this study, we explored the selenium-binding proteins in the cell lysate from the rat heart using its reactive metabolic intermediate STS and MALDI TOF-mass spectrometry. Several proteins with Cys thiol were found to be reactive with STS through the thiol-exchange reaction. The most distinctive Cys-containing protein in the cardiac cell lysate was identified as the myoglobin from a rat protein database search and tryptic fragmentation experiments. When separately examined in selenium-adequate rats, selenium-binding to the cardiac Mb was verified by the selenium-specific fluorometry (DAN method). Cardiac Mb participates in the selenium metabolism in the heart. If the cardiac $\mathrm{Mb}$ can control antioxidative selenium with the oxygen supply in defensive compensation for the oxidative damage, it may be a purposive function of $\mathrm{Mb}$. We demonstrated that selenium from STS binds to the cardiac Mb through its thiol groups. GSSeSG generated during the course of the SA metabolism would be bound to the cardiac Mb, and Mb, not only GSH, also participates in the selenium metabolism after the formation of STS in the heart. Future studies should focus on the elucidation of the physiologically possible interactions of the selenium-bound $\mathrm{Mb}$ with thiol-containing cytosolic substances. Consequently, our 
methodology using a combination of a reactive selenium metabolic intermediate and the mass spectrometric technique was effective for profiling the selenium-binding proteins and/or selenium-interactive species in the rat heart. Further investigations using PenSSeSPen as a model of biogenic STSs may uncover the variety of biologically relevant thiols in the selenium metabolism in vivo.

\section{Conflicts of interest}

The authors declare no competing fi nancial interest.

\section{References}

1 M. Rayman, Selenium and human health, Lancet, 2012, 379, 1256-1268.

2 G. V. Kryukov, S. Castellano, S. V. Novoselov, A. V. Lonanov, O. Zehtab, R. Guigó and V. N. Gladyshev, Characterization of mammalian selenoproteomes, Science, 2003, 300, $1439-1443$.

3 L. V. Papp, J. Lu, A. Holmgren and K. K. Khanna, From selenium to selenoproteins: synthesis, identity, and their role in human health, Antioxid. Redox Signaling, 2007, 9, $775-806$.

4 U. S. Department of Agriculture ARS. USDA National Nutrient Database for Standard Reference, Release 28, https://www.ars.usda.gov/ARSUserFiles/80400525/Data/SR/sr28/sr28 doc.pdf. 
5 Keshan Disease Research Group of the Chinese Academy of Medical Sciences, Observations on effect of sodium selenite in prevention of Keshan disease, Chinese Med. J., 1979, 92, 471-476.

6 J. Pei, W. Fu, L. Yang, Z. Zhang and Y. Liu, Oxidative stress is involved in the pathogenesis of Keshan disease (an endemic dilated cardiomyopathy) in China, Oxid. Med. Cell. Longev., 2013, 2013, 474203.

7 G. Flores-Mateo, A. Navas-Acien, R. Pastor-Barriuso and E. Guallar, Selenium and coronary heart disease: a meta-analysis, Am. J. Clin. Nutr., 2006, 84, 762-773.

8 S. Stranges, J. R. Marshall, M. Trevisan, R. Natarajan, R. P. Donahue, G. F. Combs, E. Farinaro, L. C. Clark and M. E. Reid, Effects of selenium supplementation on cardiovascular disease incidence and mortality: secondary analyses in a randomized clinical trial, Am. J. Epidemiol., 2006, 163, 694-699.

9 S. M. Lippman, E. A. Klein, P. J. Goodman, M. S. Lucia, I. M. Thompson, L. G. Ford, H. L. Parnes, L. M. Minasian, J. M. Gaziano, J. A. Hartline, J. K. Parsons, J. D. 3rd Bearden, E. D. Crawford, G. E. Goodman, J. Claudio, E. Winquist, E. D. Cook, D. D. Karp, P. Walther, M. M. Lieber, A. R. Kristal, A. K. Darke, K. B. Arnold, P. A. Ganz, R. M. Santella, D. Albanes, P. R. Taylor, J. L. Probstfield, T. J. Jagpal, J. J. Crowley, F. L. Jr. Meyskens, L. H. Baker and C. A. Jr. Coltman, Effect of selenium and vitamin E on risk of prostate cancer and other cancers: the selenium and vitamin E cancer prevention trial (SELECT), JAMA 2009, 301, 39-51.

10 S. J. Fairweather-Tait, Y. Bao, M. R. Broadley, R. Collings, D. Ford, J. E. Hesketh and R. Hurst, Selenium in human health and disease, Antioxid. Redox Signaling, 2011, 14, $1337-1383$. 
11 M. P. Longnecker, P. R. Taylor, O. A. Levander, M. Howe, C. Veillon, P. A. McAdam, K. Y. Patterson, J. M. Holden, M. J. Stampfer and J. S. Morris, Dietary patterns and breast cancer risk: a systematic review and meta-analysis, Am. J. Clin. Nutr., 1991, 53, $1288-1294$.

12 W. T. Self, L. Tsai and T. C. Stadtman, Synthesis and characterization of selenotrisulfide derivatives of lipoic acid and lipoamide, Proc. Natl. Acad. Sci. U. S. A., 2000, 97, 1248112486.

13 T. Lindemann and H. Hintelmann, Identification of selenium-containing glutathione S-conjugates in a yeast extract by two-dimensional liquid chromatography with inductively coupled plasma MS and nanoelectrospray MS/MS detection, Anal. Chem., $2002,74,4602-4610$.

14 M. Haratake, K. Fujimoto, M. Ono and M. Nakayama, Selenium binding to human hemoglobin via selenotrisulfide, Biochim. Biophys. Acta, 2005, 1723, 215-220.

15 M. Haratake, K. Fujimoto, R. Hirakawa, M. Ono and M. Nakayama, Hemoglobinmediated selenium export from red blood cells, J. Biol. Inorg. Chem., 2008, 13, 471-479.

16 M. Haratake, M. Hongoh, M. Miyauchi, R. Hirakawa, M. Ono and M. Nakayama, Albumin-mediated selenium transfer by a selenotrisulfide relay mechanism, Inorg. Chem., 2008, 47, 6273-6280.

17 M. Haratake, M. Hongoh, M. Ono and M. Nakayama, Thiol-dependent membrane transport of selenium through an integral protein of the red blood cell membrane, Inorg. Chem., 2009, 48, 7805-7811.

18 M. Hongoh, M. Haratake, T. Fuchigami and M. Nakayama, A thiol-mediated active membrane transport of selenium by erythroid anion exchanger 1 protein, Dalton Trans., 
2012, 41, 7340-7349.

19 P. A. Fernandes and M. J. Ramos, Theoretical insights into the mechanism for thiol-disulfide exchange, Chem. Eur. J., 2004, 10, 257-266.

20 E. Hori, S. Yoshida, M. Haratake, S. Ura, T. Fuchigami and M. Nakayama, An effective method for profiling the selenium-binding proteins using its reactive metabolic intermediate, J. Biol. Inorg. Chem., 2015, 20, 781-789.

21 M. P. Bansal, R. G. Cook, K. G. Danielson and D. Medina, A 14-kilodalton selenium-binding protein in mouse liver is fatty acid-binding protein, J. Biol. Chem., 1989, 264, 13780-13784.

22 G. D. Lopaschuk, J. R. ussher, C. D. L. Folmes, J. S. Jaswal and W. C. Stanley, Myocardial fatty acid metabolism in health and disease, Physiol. Rev., 2010, 90, $207-$ 258.

23 S. Boudina and E. D. Abel, Physiology, 2005, 21, 250-258.

24 M. Haratake, M. Ono and M. Nakayama, Penicillamine-selenotrisulfide as an selenium-source in mice, J. Health Sci., 2004, 50, 366-371.

25 O. H. Lowry, N. J. Resebrough, A. L. Farr and R. J. Randall, Protein measurement with the Folin phenol reagent, J. Biol. Chem., 1951, 193, 265-275.

26 T. M. T. Sheehan and M. Gao, Simplified fluorometric assay of total selenium in plasma and urine, Clin. Chem., 1990, 36, 2124-2126.

27 G. L. Ellman, Arch. Biochem. Biophys., A colorimetric method for determining low concentrations of mercaptans, 1958, 74, 443-450.

28 L. Flohe and W. A. Gunzler, Assays of glutathione peroxidase, Methods Enzymol., 1984, $105,114-120$. 
29 M. Haratake, J. Takahashi, M. Ono and M. Nakayama, An assessment of Niboshi (a processed Japanese anchovy) as an effective food source of selenium, J. Health Sci., $2007, \mathbf{5 3}, 457-463$.

30 P. R. Hoffman, S. C. Hoge, P. Li, F. W. Hoffman, A. C. Hashimoto and M. J. Berry, The selenoproteome exhibits widely varying, tissue-specific dependence on selenoprotein $\mathrm{P}$ for selenium supply, Nucleic Acids Res., 2007, 35, 3963-3973.

31 G. P. Guyton, K. S. Stanek, R. C. Schneider, P. W. Hochachka, W. E. Hurford, D. G. Zapol, G. C. Liggins and W. M. Zapol, Myoglobin saturation in free-diving Weddell seals, J. Appl. Physol., 1995, 79, 1148-1155.

32 A. E. Herrera and H. Lehmann, Primary structure of human myoglobin, Nature New Biol., 1971, 232, 149-152.

33 M. Maj, Y. Oh, K. Park, J. Lee, K. Kwak and M. Cho, Vibrational dynamics of thiocyanate and selenocyanate bound to horse heart myoglobin, J. Chem. Phys., 2014, 140, 235104.

34 H. Imai and Y. Nakagawa, Biological significance of phospholipid hydroperoxide glutathione peroxidase (PHGPx, GPx4) in mammalian cells, Free Radic. Biol. Med., $2003,34,145-169$.

35 H. Imai, F. Hirao, T. Sakamoto, K. Sekine, Y. Mizukura, M. Saito, T. Kitamoto, M. Hayasaka, K. Hanaoka and Y. Nakagawa, Early embryonic lethality caused by targeted disruption of the mouse PHGPx gene, Biochem. Biophys. Res. Commun., 2003, 305, $278-286$.

36 L. J. Yant, Q. Ran, L. Rao, H. V. Remmen, T. Shibatani, J. G. Belter, L. Motta, A. Richardson and T. A. Prolla, The selenoprotein GPX4 is essential for mouse development 
and protects from radiation and oxidative damage insults, Free Radic. Biol. Med., 2003, 34, 496-502. 
Table 1 Changes in selenium amount bound to proteins in the rat cardiac cell lysate accompanying the reaction with increasing PenSSeSPen concentration. Reaction time: 30 min. Reaction temperature: $37^{\circ} \mathrm{C}$. Protein thiol amount in the rat heart cell lysate: $20.98 \pm$ $0.57 \mu \mathrm{mol} \mathrm{g}{ }^{-1}$-protein. Data are mean \pm standard error. $(n=4-6)$

PenSTSPen concentration (mM)

Selenium amount ( $\mu \mathrm{mol} \mathrm{g}{ }^{-1}$-protein)

0

$0.037 \pm 0.002$

0.01

$5.592 \pm 1.06$

0.05

$14.75 \pm 1.89$

0.1

$19.16 \pm 2.45$

0.2

$20.13 \pm 0.50$ 
Table 2 Observed molecular mass gain in MALDI TOF-mass spectra of rat heart cell lysate before and after reaction with NEM and PenSSeSPen. n. d. : not detected. NEM and PenSSeSPen concentrations used: $1 \mathrm{mM}$ and $50 \mu \mathrm{M}$. Reaction temperature: $37^{\circ} \mathrm{C}$. Reaction time: 10 min.

\begin{tabular}{|c|c|c|}
\hline $\begin{array}{l}\text { Original peak in } m / z \\
\text { before reaction (a) }\end{array}$ & $\begin{array}{l}\text { Peak in } m / z \text { after reaction } \\
\text { with NEM } \\
(\mathrm{a}+125,+250 \text { and }+375)\end{array}$ & $\begin{array}{l}\text { Peak in } m / z \text { after reaction } \\
\text { with PenSSeSPen } \\
(\mathrm{a}+226)\end{array}$ \\
\hline 3636.60 & 3762.16 & 3862.93 \\
\hline 4047.33 & 4171.75 & 4272.47 \\
\hline 4183.43 & 4308.33 & 4410.52 \\
\hline 11241.22 & 11366.10 & n. d. \\
\hline 12349.24 & $12473.14,12598.36,12723.09$ & n. d. \\
\hline 14997.70 & 15122.17 & n. d. \\
\hline 15041.67 & 15167.76 & n. d. \\
\hline 15154.37 & $15406.15,15529.85$ & n. d. \\
\hline 15198.38 & $15324.39,15449.47,15574.23$ & 15425.52 \\
\hline 15837.86 & 16088.14 & n. d. \\
\hline 17026.59 & 17152.02 & 17252.65 \\
\hline 17235.78 & 17361.67 & 17462.42 \\
\hline 17814.35 & 17939.08 & 18041.06 \\
\hline
\end{tabular}




\section{Figure legends}

Fig. 1 Thiol-exchange reaction of PenSSeSPen with a protein thiol.

Fig. 2 MALDI TOF-mass spectrometric ananlysis of reaction of NEM and PenSSeSPen with $m / z 17026$ species. (A) Before reaction, (B) after reaction with $1 \mathrm{mM} \mathrm{NEM,} \mathrm{(C)} \mathrm{after}$ reaction with $50 \mu \mathrm{M}$ PenSSeSPen. Reaction medium: $10 \mathrm{mM}$ phosphate buffer ( $\mathrm{pH}$ 7.4). Reaction temperature: $37^{\circ} \mathrm{C}$. Reaction time: $10 \mathrm{~min}$.

Fig. 3 MALDI TOF-mass spectrometric analysis of reaction of PenSSeSPen with $\mathrm{m} / z 17026$ species with increasing PenSSeSPen concentration. (A) Before reaction, (B) after reaction with $10 \mu \mathrm{M}$ PenSSeSPen, (C) after reaction with $50 \mu \mathrm{M}$ PenSSeSPen, (D) after reaction with $100 \mu \mathrm{M}$ PenSSeSPen. Reaction medium: $10 \mathrm{mM}$ phosphate buffer (pH 7.4). Reaction temperature: $37^{\circ} \mathrm{C}$. Reaction time: $10 \mathrm{~min}$.

Fig. 4 Amino acid sequence of rat myoglobin. Protein Information Resource (PIR, http://www-nnbrf.geogetown.edu/pirwww/): Myoglobin-Rattus norvegicus (Rat).

Fig. 5 Purification of rat cardiac Mb. (A) MALDI TOF-mass spectrum of rat cardiac cell lysate before purification. Arrow indicates the peak of rat $\mathrm{Mb}$ at $m / z$ 17026.5. SDS-PAGE of (a) before and (b) after purification.

Fig. 6 MALDI TOF-mass spectrometric analysis of the reactivity of selenious acid (SA) 
with $\mathrm{m} / z 17026$ species in the rat cardiac cell lysate. (A) Before reaction, (B) after reaction with $0.1 \mathrm{mM} \mathrm{SA},(\mathrm{C})$ after reaction with $0.1 \mathrm{mM}$ SA and $0.05 \mathrm{mM} \mathrm{GSH}$. Reaction time: $3 \mathrm{~h}$, reaction temperature: $37{ }^{\circ} \mathrm{C}$. Dotted lines were drawn at $\mathrm{m} / z 17026$ (free $\mathrm{Mb}$ ) and 17411 (SeSG-adduct).

Fig. 7 (A) Selenium concentrations of heart in rat and (B) cellular GPx activity for hydrogen peroxide and tert-butyl hydoroperoxide $(t \mathrm{BHP})$ in the cardiac cell lysate. non-administered (regular diet only), $\square$ SA-administered. Selenious acid in saline was orally administered to 6-week old male mice at a dose of $1.5 \mu \mathrm{gSe} \mathrm{\textrm {kg } ^ { - 1 } \text { -body weight day }}{ }^{-1}$ for two weeks. Data express mean and SD $(n=5) . \quad *$ Different from the non-administered group, $p<0.05$. 
Fig. 1<smiles>CC(C)(SS[Se]SC(C)(C)[C@@H](N)C(=O)O)[C@@H](N)C(=O)O</smiles><smiles>CCCCC(C)[SbH]CCC</smiles><smiles>CC(=O)SS[Se]SC(C)(C)[C@H](N)C(=O)O</smiles> 
Fig. 2

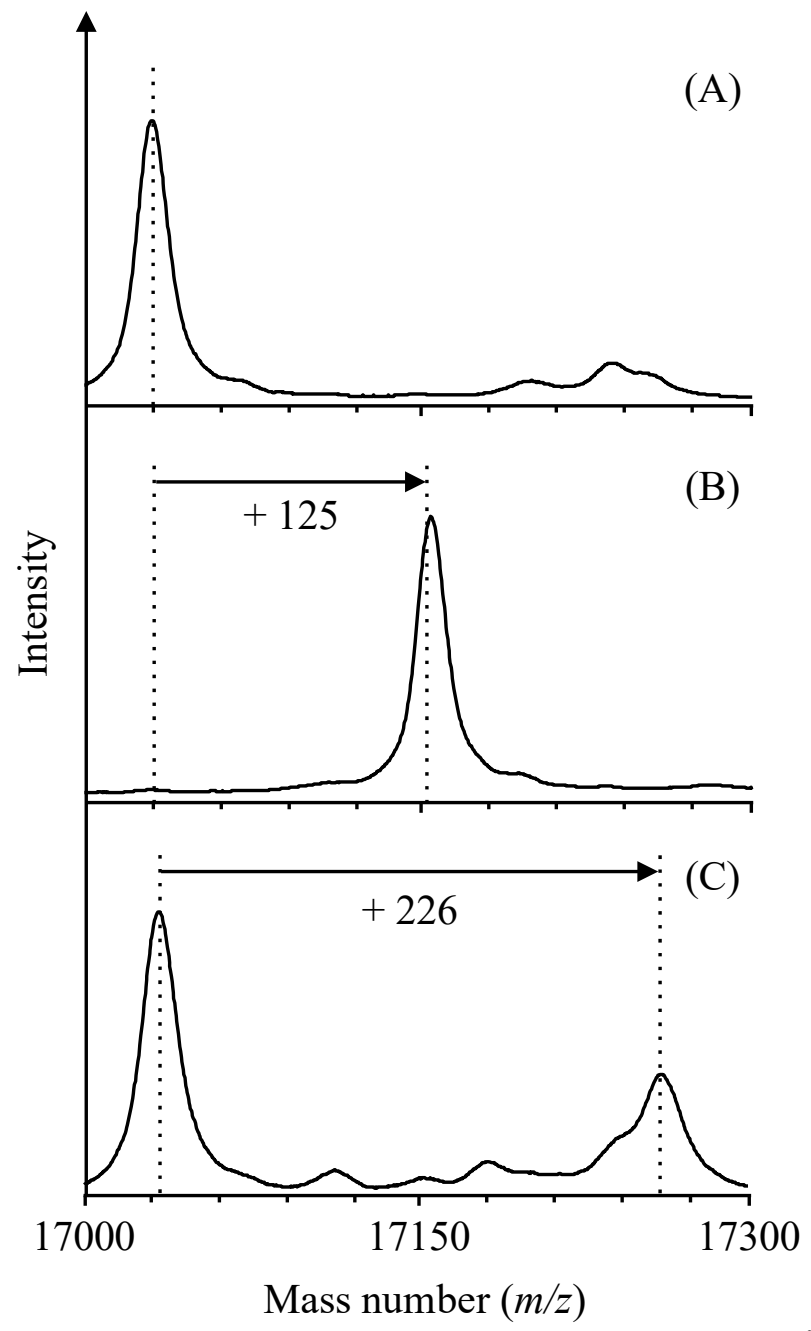


Fig. 3

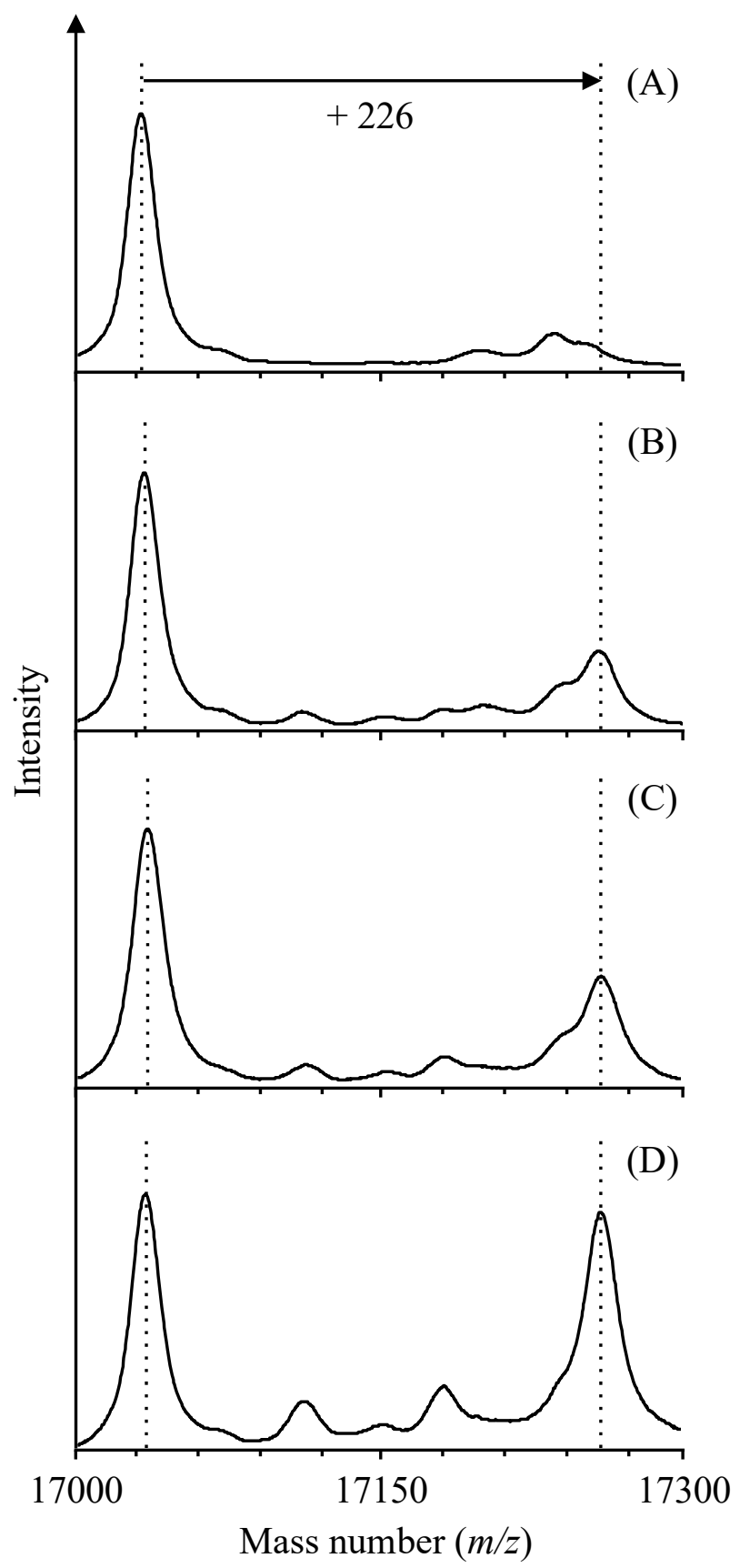


Fig. 4

1

Gly Leu Ser Asp Gly Glu Trp Gln Met Val Leu Asn Ile Trp Gly Lys Val Glu Gly Asp 21

Leu Ala Gly His Gly Gln Glu Val Leu Ile Ser Leu Phe Lys Ala His Pro Glu Thr Leu 41

Glu Lys Phe Asp Lys Phe Lys Asn Leu Lys Ser Glu Glu Glu Met Lys Ser Ser Glu Asp $61 \quad 66$

Leu Lys Lys His Gly Cys Thr Val Leu Thr Ala Leu Gly Thr Ile Leu Lys Lys Lys Gly 81

Gln His Ala Ala Glu Ile Gln Pro Leu Ala Gln Ser His Ala Thr Lys His Lys Ile Pro 101

Val Lys Tyr Leu Glu Phe Ile Ser Glu Val Ile Ile Gln Val Leu Lys Lys Arg Tyr Ser 121 131

Gly Asp Phe Gly Ala Asp Ala Gln Gly Ala Met Ser Lys Ala Leu Glu Leu Phe Arg Asn 141

Asp Ile Ala Ala Lys Tyr Lys Glu Leu Gly Phe Gln Gly 
Fig. 5
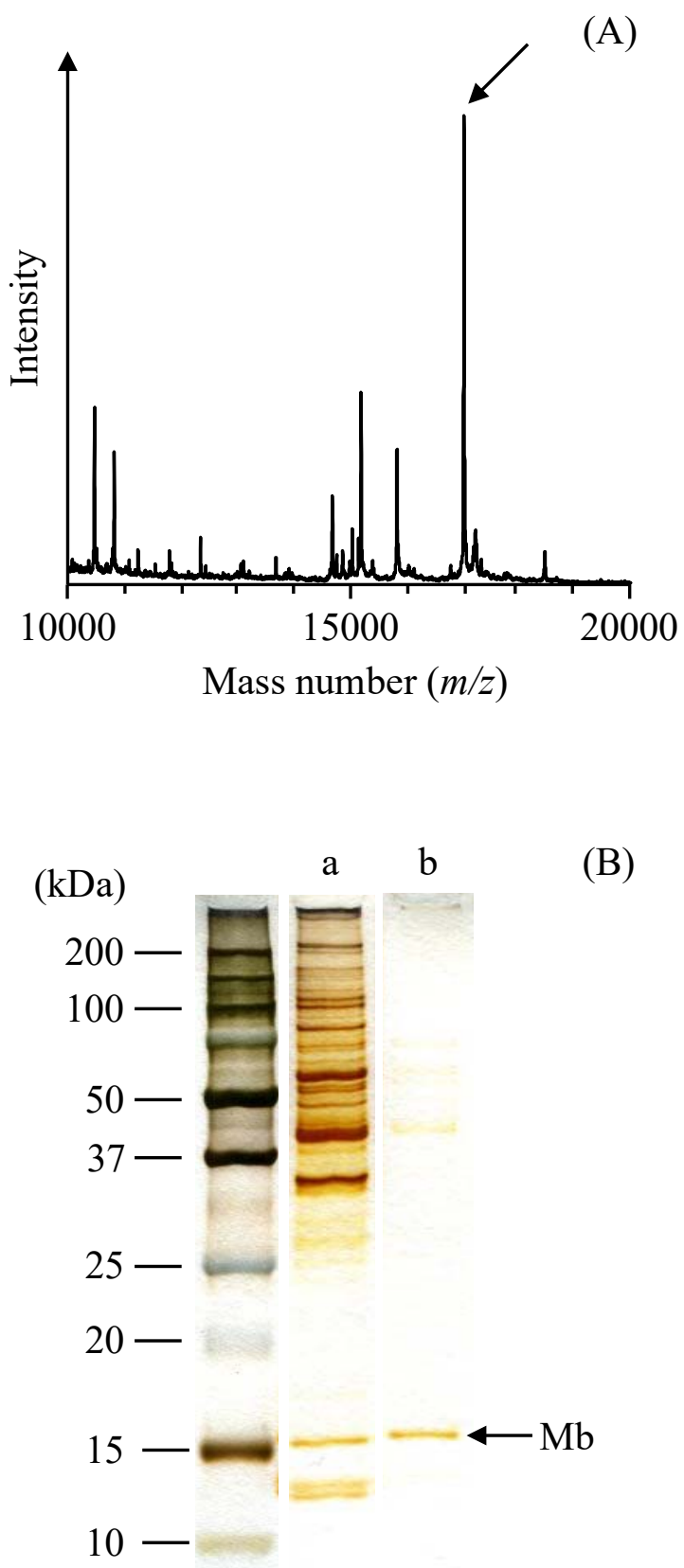
Fig. 6

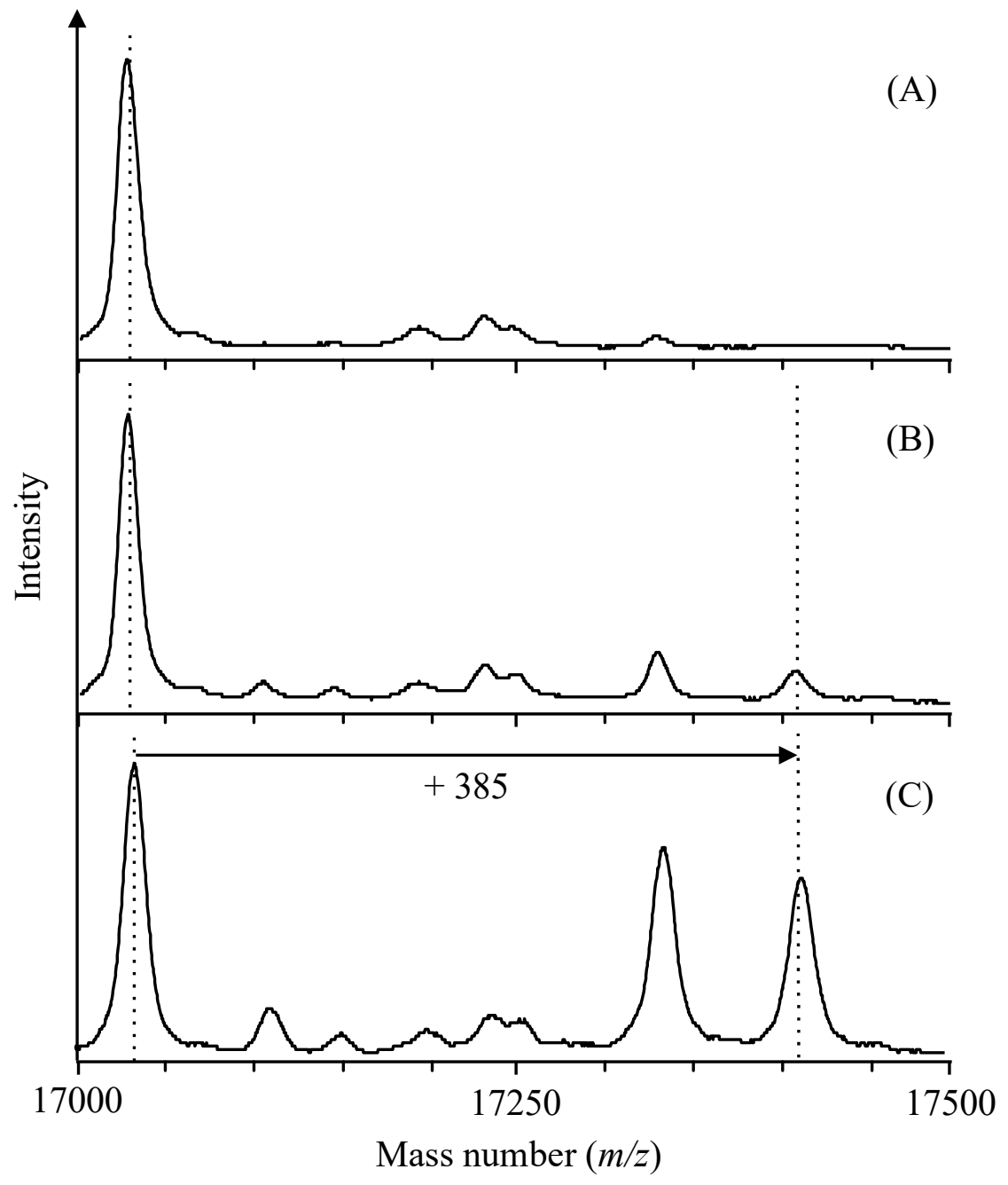


Fig. 7

(A)

\begin{tabular}{ll}
\hline Treatment & $\begin{array}{l}\text { Selenium concentration } \\
\left(\mu g S e g^{-1} \text {-heart }\right)\end{array}$ \\
\hline $\begin{array}{l}\text { Non-administered } \\
\text { (regular diet only) }\end{array}$ & $0.26 \pm 0.056$ \\
SA-administered & $0.51 \pm 0.16$
\end{tabular}

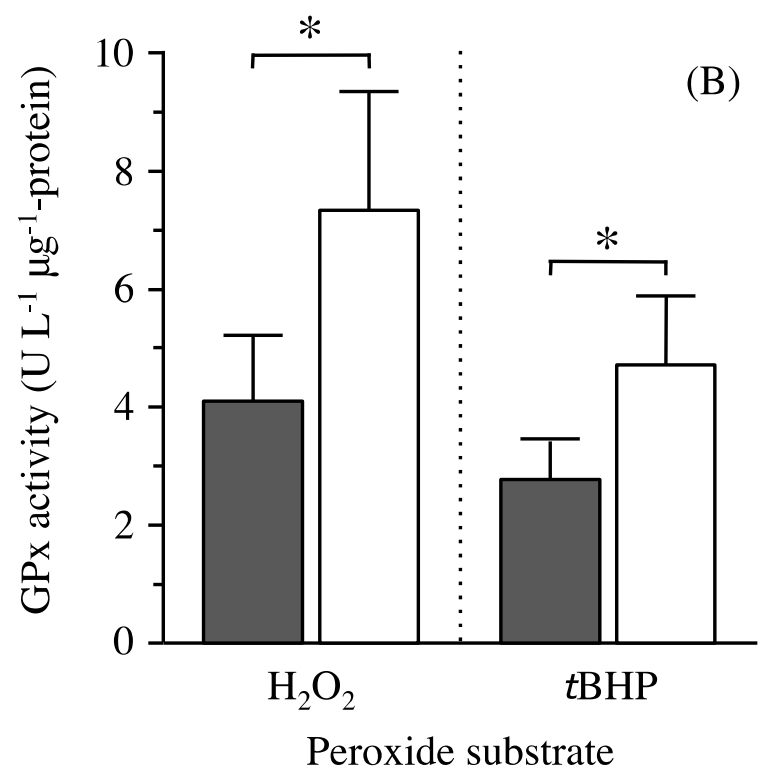

